\title{
Amniotic sludge and short cervix as inflammation and intraamniotic infection markers
}

\begin{abstract}
Infection is the leading cause of preterm labor. Short cervix and amniotic sludge are related with an increased risk of preterm delivery and intra amniotic infection. The risk of infection is inversely proportional to sonographic cervical length and weeks of gestation at diagnosis. Antibiotic treatment with the agent and adequate dose may delay the gestational age at birth, even more if it is performed before the onset of symptoms.
\end{abstract}

Volume 7 Issue 2 - 2017

\author{
Javier Perez Pedregosa,' M Coronado Ruiz, ${ }^{2}$ \\ T Bueso Medina, ${ }^{2}$ A Galvez Rascın, ${ }^{2}$ J del \\ Gallo, ${ }^{2}$ J Lazaro de la Fuente, ${ }^{3}$ MJ Teran \\ Alonso $^{2}$ \\ 'Department of Obstetrics and Gynecology, Universidad \\ Francisco de Vitoria, Spain \\ ${ }^{2}$ Department of Obstetrics and Gynecology, Hospital \\ Universitario Sanitas La Moraleja, Spain \\ ${ }^{3}$ Department of Obstetrics and Gynecology, Hospital \\ Universitario Ram?n y Cajal, Spain
}

\begin{abstract}
Correspondence: Javier Perez Pedregosa, Fetal Medicine Unit, Department of Obstetrics and Gynecology, Hospital Universitario Sanitas La Moraleja, Universidad Francisco de
\end{abstract} Vitoria, Madrid, Spain, Email obsgyn@hotmail.com

Received: October 10, 2016 | Published: May 25, 2017
Abbreviations: FIRS, Fetal Inflammatory Response Syndrome; PPROM, Preterm Premature Rupture of Membranes; PL, Preterm Labor; MIAC, Microbial Invasion of the Amniotic Cavity; SI,Sterile Inflammation

\section{Introduction}

Preterm delivery is a complex syndrome with multiple causes but current evidence shows that intra-amniotic infection is the leading one. Pathogenic bacteria may ascend from the lower reproductive tract and the resulting inflammation leads to preterm labor, rupture of membranes and birth. The different stages of progression and colonization of the amniotic membranes, passage of germs into the cavity and fetal swallowing of these and their inflammation products have been described by different authors. The arrival of germs to the amniotic cavity by haematogenous route is also possible but much less frequent. This process of ascent and colonization of the membranes by different microorganisms is generally slow and is influenced by factors such as type and inoculum of agents involved, and immune conditions of the host. The fetal response in a final stage could also be determinant for the onset of labor and the risk of fetal involvement with the appearance of a clinical picture known as fetal inflammatory response syndrome (FIRS). The aim of this study was to define the frequency of the intra-amniotic infection in patients with short cérvix and/or amniotic fluid (AF) "sludge". The pregnancy outcome and his relationship with preterm delivery (PD) and/or preterm premature rupture of membranes (PPROM) was evaluated.

\section{Material and methods}

This prospective and retrospective study analyzed cases from November 2012 to January 2015. We included singleton pregnancies between 16 and 32 weeks of gestation with a cervical length $\leq 25$ $\mathrm{mm}$ and/or the presence of amniotic sludge. The cervical length was performed in every case by transvaginal ultra sonography with a 5-7.5
MHz probe and empty bladder. We performed 3 measurements and the shortest was chosen. The diagnosis of AF "sludge" was made after the finding of dense aggregates of particulate matter in the amniotic fluid close to the internal cervical os. We excluded patients with PPROM, preterm labor (PL), cervical dilatation $\geq 2 \mathrm{~cm}$ and clinical criteria of chorio amnionitis at the time of diagnose.

In all cases transabdominal amniocentesis was offered and we obtained informed consent in all cases that it was performed. The diagnosis of intra-amniotic infection (MIAC, Microbial Invasion of the Amniotic Cavity) was performed after the presence of bacteria in a culture, positive Gram staining or multiplex PCR and for Mycoplasma hominis in AF obtained by transabdominal amniocentesis. PCR multiplex included: Chlamydia T, Neisseria G, Mycoplasma G, Trichomonas V, Gardnerella V, Ureaplasma U\&P, and HSV I-II.

In those patients with more than 30 white blood cells (WBC)/ $\mathrm{mm} 3 \mathrm{and} /$ or glucose $<15 \mathrm{mg} / \mathrm{dl}$, and culture and PCR negative, the diagnosis was sterile inflammation $(S I)$ and the treatment was similar.

All pregnant women with positive results in $\mathrm{AF}$ or those with $\mathrm{AF}$ "sludge" that renounced amniocentesis were admitted and treated with intravenous azithromycin $1 \mathrm{gr}$ per day for seven days. We added other antibiotic agents in the cases were recommended by the result of culture and antibiotic sensitivity test. In one of the patients the treatment was completed with oral azithromycin due to a local cutaneous reaction during intravenous administration.

All patients with short cervix were treated with vaginal natural micronized progesterone (200 mg per day) and in 7 cases, an Arabin cervical pessary was inserted during evolution.

\section{Results}

22 pregnant women had the inclusion criteria. 10 (45.4\%) were completely asymptomatic at diagnosis. Between symptomatic 
patients, $4(33.3 \%)$ had self-limited and low volume metrorrhagia in the previous days, $3(25 \%)$ had irregular dynamic without preterm labor criteria, $2(16.6 \%)$ had a intense feeling of supra pubic weight and 3 patients had genital bleeding and irregular dynamic.

The mean cervical length was $11.3 \mathrm{~mm}$ at diagnosis (range 2.9-31 $\mathrm{mm})$. In 14 (63.6\%) cases the presence of AF "sludge" was observed. The mid cervical length in patients with amniotic "sludge" was equal to that of patients with short cervix isolated, despite including the group patients with $\mathrm{AF}$ "sludge" gestations with any cervical length. 15 pregnant women accepted the realization of amniocentesis to dismiss subclinical infection. In 8 cases $(53.3 \%)$ we had a positive culture, Gram staining or PCR, establishing the diagnosis of MIAC. Genital Mycoplasmas (M. Hominis and U. Urealiticum) were the most commonly isolated infectious agents. SI was diagnosed in 6 cases $(40 \%)$ by the presence of more than $30 \mathrm{WBC} / \mathrm{mm} 3$ without identifying any infectious agent. Only one case was negative, and this pregnant woman gave birth by cesarean section for breech presentation at 36 weeks after PPROM. Among the patients with MIAC the medium time of prolongation of the pregnancy was 21 days (range 1-108) vs. 60.5 days (range 1-133) in those with SI. 9 (40.9\%) of the patients reached the end of gestation. $3(13.6 \%)$ requested legal abortion after an early PPROM $(<23$ weeks) and anhydramnios. There were $5(22.7 \%)$ with fetal demise $(<25$ weeks). $5(22.7 \%)$ remaining patients had a preterm delivery with a mean gestational age at birth of 29 weeks (range 25-
32). Of the 22 patients included, 14 had AF "sludge" diagnosis, in 12 the amniocentesis was performed, and in all cases the $\mathrm{AF}$ was positive (8 MIAC and $4 S I$ ). Among the 14 patients with MIAC or SI, only 4 $(28.6 \%)$ reached the term (Table 1$)$.

\section{Table I Results of Amniocentesis}

\begin{tabular}{llll}
\hline Performed amniocentesis (n=I5) & MIAC & Si & Normal \\
\hline Short cervix (3) & & $\mathbf{6 6 . 7 0 \%}$ & $\mathbf{3 3 . 3 0 \%}$ \\
Asymptomatic (I) & 0 & $\mathrm{I}(100 \%)$ & 0 \\
Symptomatic(2) & 0 & $\mathrm{I}(50 \%)$ & $\mathrm{I}(50 \%)$ \\
Short cérvix + Sludge (12) & $\mathbf{6 6 . 7 0 \%}$ & $\mathbf{3 3 . 3 0 \%}$ & \\
Asymptomatic (5) & $4(80 \%)$ & $\mathrm{I}(20 \%)$ & 0 \\
Symptomatic (7) & $4(57.1 \%)$ & $3(42.9 \%)$ & 0
\end{tabular}

Microbiological study of the placenta, membranes and umbilical cord was performed in 11 cases. The culture was positive in 10 of them, usually with several different infectious agents to those isolated in the amniotic fluid in those patients with previous amniocentesis and treated with intravenous azitromicine. Three were performed in patients who declined amniocentesis, and in all cases it was positive for various microorganisms, one of them in a patient $19+3$ weeks symptomatic, with $11 \mathrm{~mm}$ of cervical length and AF sludge, which reached the end of the gestation after antibiotic treatment, and 2 symptomatic patients who progressed quickly after initial findings and had fetal death after early PPROM (Table 2).

Table 2 Delivery results according to the presence of sludge or not

\begin{tabular}{|c|c|c|c|c|}
\hline & $\begin{array}{l}\text { Fetal Demise } \\
\text { (<25 Weeks) }\end{array}$ & $\begin{array}{l}\text { preterm Delivery } \\
\text { (25-34 Weeks) }\end{array}$ & $\begin{array}{l}\text { preterm Delivery } \\
\text { (34-37 Weeks) }\end{array}$ & $\begin{array}{l}\text { term Delivery } \\
\text { (>37 Weeks) }\end{array}$ \\
\hline Short cervix (8) & $-12.50 \%$ & & $-25 \%$ & $-62.50 \%$ \\
\hline Asymptomatic (5) & 0 & 0 & 0 & $5(100 \%)$ \\
\hline Symptomatic (3) & I (33.3\%) & 0 & $2(66.7 \%)$ & 0 \\
\hline Short cervix + Sludge (14) & $-50 \%$ & $-14.20 \%$ & $-7.10 \%$ & $-28.50 \%$ \\
\hline Asymptomatic (5) & $3(60 \%)$ & I (20\%) & 0 & I (20\%) \\
\hline Symptomatic (9) & $4(44.4 \%)$ & $\mathrm{I}(\mathrm{II} . \mathrm{I} \%)$ & I (II.I\%) & $3(33.3 \%)$ \\
\hline
\end{tabular}

\section{Discussion}

PD is one of the major global health problems and its incidence is stable or even rising in some regions in the last decades despite the great efforts and resources dedicated to change this tendency. Morbidity and mortality is inversely proportional to gestational age at birth. The improvements in survival rate achieved in recent years are mainly due to specialized care in intensive neonatal care units. A preterm birth is a burden for the individual, their families and society, with possible repercussions even during adulthood. ${ }^{1}$ PL has been defined as a major syndrome which is reached by different ways. The infection has a main role and depending on the clinical presentation may reach $40 \%$ of cases. Of all the known causes of PL, this is the only one with a well described fisiopathological and molecular base, ${ }^{2}$ although the proper management of this cases remains controversial and not uniform at all in different Centers. In asymptomatic patients with a short cervix, the likelihood of infection is inversely proportional to the cervical length and gestational age at diagnosis. ${ }^{3}$ The frequency of intra-amniotic infection is even higher in patients with PPROM and PL. However, the etiological role of intraamniotic infection is not considered in most Centers, and patient management is mainly based on symptomatic treatment with tocolytic drugs or therapeutic measures to try to stop cervical changes (progesterone, cerclage, pessary). In the medical era of individualized treatment of many diseases, we still have a very similar treatment to all those patients at risk of PL. Maybe this is the main reason for our great failure in the old attempt in the last decades to reduce spontaneous preterm birth. The sonographic diagnosis of a short cervix is a powerful predictor of spontaneous $\mathrm{PD}$, however, neither the cause or the proper management has been determined. ${ }^{4}$ AF "sludge" has been defined as an echogenic image formed by dense aggregates of particulate matter in the amniotic fluid close to the internal cervical os.

This particles could represent germs surrounded by biofilms that would hinder neutralization of germs by the antibiotics. ${ }^{5-6}$ AF"Sludge"should not be confused with dense aggregates of fetal vermis found sometimes in normal pregnancies which disintegrates after gentle pressure with the transvaginal probe.

The finding of both markers (short cervix \& amniotic "sludge") is a risk factor for MIAC, SI, PPROM, $P D$ and fetal death in asymptomatic patients and in those with PL. Preterm babies born in this clinical situation could have an increased risk of cerebral palsy when compared with other of the same gestational age without intraamniotic infection. ${ }^{7}$ In our opinion, the presence of amniotic "sludge" would be a more advanced stage of the infection, and in those of our cases that were subjectively described with a bigger AF "sludge" volume (not always associated with increased cervical shortening), the pregnancy outcome was worse, with very limited effect of antibiotic treatment. Only 5 of the 14 patients in our series with AF "sludge" reached the end of gestation. Multiple published studies that relate, in singleton pregnancies, short cervix with $M I A C$ and adverse pregnancy outcome, have been made in two different clinical settings. 
On one side PL patients and in other asymptomatic patients, or at least without clinical criteria of PL.

In a multicenter study, Gomez \& Romero ${ }^{8}$ quantified the risk of intra-amniotic infection for each gestational age in singleton pregnancies between 22 and 34 weeks with intact membranes and PL based on cervical length. ${ }^{8}$ For example, in a pregnant woman with PL at 28 weeks with a sonographic cervical length of $10 \mathrm{~mm}$, they found an estimated risk of MIAC of $24 \%$ and $12 \%$ for the same patient with $20 \mathrm{~mm}$ of cervical length. This information is of great useful to the management and prognosis information and in some cases, depending on gestational age, it could even be better to finish the pregnancy to remove the fetus from a hostile environment.

In 47 asymptomatic patients between 14-24 weeks with short cervix $(\leq 15 \mathrm{~mm})$, Romero et al. ${ }^{9}$ found a SI rate of $22.2 \%$ and $4.3 \%$ of MIAC. For the diagnosis of inflammation they studied methyl metalloproteinase 8 (MMP-8) in AF whose sensitivity may be higher than the WBC count. The rate of PD a week after the amniocentesis was $40 \%$ in patients with $M I A C$ and $5.7 \%$ in patients with inflammation.

Our findings were very similar with a significant difference in the time to delivery after the diagnosis in cases with $S I$ vs. those with infection. The prognosis was also poor in our cases with a high number of leukocytes and negative culture or PCR. Those cases would reflect limitations of microbiological techniques in a bactericide medium as amniotic fluid.

In some of our cases the microbiological agents found in the membranes, placenta and umbilical cord were different than those previously found in the amniotic fluid, with more than one bacteria found in most of them. This finding could reflect the polymicrobial origin of the disease and the partial efficacy of antibiotic treatment or even different stages of the process with an initial infection for genital mycoplasmas in most of the cases that allow the posterior invasion of more aggressive bacteria (E. Colli, Enterobacter...)

In our case series, we used the glucose and WBC count in amniotic fluid to define the presence of inflammation. This is easy to do, even though their sensitivity could be lower than other markers such as interleukin (IL) 6 and 11. We use a cut off point of 30 leuc/mm3, others authors use a higher number $(50 \mathrm{leuc} / \mathrm{mm} 3)$ to reduce the false positive rate. As previously said, Vaisbuch $\mathrm{E}^{9}$ reported higher sensitivity for the diagnosis of SI using MMP-8 in AF and new promising markers and proteomic studies will be studied in the near future to increase the detection rate of difficult cases or those at an earlier stage.

The PCR also has a high sensitivity for the detection of genital mycoplasmas but cultures may be false negative due sometimes by the bactericide effect of the AF. 5 of our cases with very high levels of leukocytes, highly suggestive of infection, had negative cultures. After birth, microbiological cultures of placenta and/or membranes were positive in all, which confirms the limitation of the amniotic fluid study, but otherwise we can't prove that the infection has occurred later, because many of these pregnant women had a short cervix, which would facilitate the rise and colonization of germs.

Given the agents most frequently found in the AF (genital mycoplasmas), the ascendant via would be the main way by the microorganisms access into the amniotic cavity making an inflammatory reaction in an intra-amniotic and choriodecidual level, which leads the release of proinflammatory markers (IL, tumor necrosis factors ...), the complement activation and the release of prostaglandins and others and finally causes the well known clinical manifestations whose order and synchronicity are not always the same in each case; cervical changes, PPROM, uterine contractions, and in un favorable cases, the onset of labor. ${ }^{2}$

Although still unclear in all its aspects, the fetus could have an important role in this situation. The FIRS is the reactive and nonspecific clinical presentation to different noxa, the main one is the intra-amniotic infection, and it probably means an advanced stage after the presence of and untreated infection. Probably his severity is different according to the germ involved, the gestational age when it occurs and other unknown maternal and fetal factors. In this clinical situation, the fetus could stimulate the onset of labor with the release of proinflammatory substances and as it's has been demonstrated is more vulnerable to infection with an increased risk of complications in many systems and organs, mainly lung, and a higher rate of neurological complications after birth. ${ }^{7-10}$ The diagnosis of FIRS is only possible through cordocentesis (IL-6 and other markers determination) or postnatal study of the umbilical cord to confirm the presence of infection (funisitis) as previously described. ${ }^{10}$ For some authors, the presence of FIRS would indicate the termination of pregnancy regardless of gestational age. In none of our cases of MIAC / SI we perform cordocentesis because a new puncture in this high risk pregnancies increases the morbidity and proper management is not clear. Only in one patient we performed a new amniocentesis to verify the absence of infection after a first positive culture for Mycoplasma hominis treated with azithromycin, and it was indicated due to the progression of cervical shortening and previous history of early PPROM and fetal death after infection. The result was negative and the patient reached the end of gestation. It is not established the role of antibiotic treatment in patients with SI or subclinical MIAC. The effectiveness of the treatment is difficult to prove given the limitations of ethical studies in humans, because it is not possible to perform an amniocentesis and in case of positive result not to treat the patient, and we have to assume that this study will never be performed.

It is not clear what is the best antibiotic agent to reach the effective concentrations in the amniotic fluid and the fetal blood. Most of the most experienced groups employ intravenous azithromycin at high doses ( 1 g every 24 hours for 7 days). ${ }^{11}$ All our patients with a positive result were treated at least with this agent.

In a multicenter study ${ }^{3}$ with 152 asymptomatic pregnancies between 14-24 weeks with a cervical length $<25 \mathrm{~mm}, 57$ patients accepted the realization of amniocentesis. It was positive in $5(9 \%) .4$ of them by Ureaplasma urealyticum. After the treatment with intravenous azithromycin, 3 had a term birth and the other gave birth $<32$ weeks, although 7 weeks after the diagnosis. They repeat amniocentesis in all cases after antibiotic treatment. All cultures had become negative, although the pregnant who had a preterm birth maintained a high rate of leukocytes in the second puncture that could reflect a false negative result of the culture.

In a classic study published in 1992 , Gray et al. ${ }^{12}$ found between 2461 second trimester genetic amniocentesis, $9(0.37 \%)$ positive Ureaplasma urealyticum cultures. None of the pregnant women were treated with antibiotics. One patient finalized the gestation voluntarily by malformation, 6 had preterm births and 2 had PL within 4 weeks after the puncture. The limitations of this study include the absence of a control group but it can be used to show the natural history of the untreated disease. In conclusion, in singleton pregnancies with intact membranes, the presence of a short cervix and/or AF "sludge", is a marker of MIAC/SI and adverse pregnancy outcome with an increased risk of PPROM, late abortion, fetal death or PD. Antibiotic treatment 
could prolong gestation in selected cases. Although infections may be caused by various agents, genital mycoplasmas (M. Hominis \& U. Urealiticum) are the most frequently isolated. The ethical limitations that govern human experimentation, make not possible to perform a prospective and randomized trial study to demonstrate the benefit of treatment. We should look for infection in this clinical situations and treat it in case of a positive result. To do nothing is no longer an option.

\section{Acknowledgments}

None.

\section{Conflicts of interest}

None.

\section{References}

1. Roger Robinson. The fetal origins of adult disease. No longer just a hypothesis and may be critically important in south Asia. BMJ. 2001;322-375.

2. Romero R, Espinoza J, Gonçalves LF, et al. Inflammation in preterm and term labour and delivery. Seminars Fetal Neonatal Med. 2006;11(5):317-326.

3. Hassan S, Romero R, Hendler I, et al. A sonographyc short cérvix as the only clinical manifestation of intra-amniotic infection. $J$ Perinat Med. 2006;34(1):13-19.

4. Iams JD, Goldenberg RL, Meis PJ, et al. The length of the cervix and the risk of spontaneous premature delivery. The New England Journal of Medicine. 1996;334:567-573.
5. Romero R, Kusanovic JP, Espinoza J, et al. What is a amniotic fluid "sludge"? Ultrasound Obstet Gynecol. 2007;30(5):793-798.

6. Kusanovic JP, Espinoza J, Romero R, et al. Clinical significance of the presence of amniotic fluid "sludge" in asymptomatic patients at high risk for spontaneous preterm delivery. Ultrasound Obstet Gynecol. 2007;30(5):706-714.

7. Wu YW, Collard J M. Chorioamnionitis as a risk factor for cerebral palsy. A meta-analysis. JAMA. 2000;284(11):1417-1424

8. Gomez R, Romero R, Nien JK, et al. A short cervix in women with preterm labor and intact membranes: A risk factor for microbial invasion of the amniotic cavity. American Journal of Obstetrics and Gynecology. 2005;192(3):678-689.

9. Vaisbuch E, Hassan S, Mazahi Tovi S, et al. Patients with an asymptomatic short cervix $(\leq 15 \mathrm{~mm})$ have a high rate of subclinical intraamniotic inflammation: implications for patient counceling. $\mathrm{Am} J$ Obstet Gynecol. 2010;202(5):433.e1-433.e8.

10. Gotsch F, Romero R, Kusanovic JP, et al. The fetal inflamatory response síndrome. Clin Obstet Gynecol. 2007;50(3):652-683.

11. Grigsby PL, Novy MJ, Sadowsky DW, et al. Maternal azithromycin therapy for Ureaplasma intraamniotic infection delays preterm delivery and reduces fetal lung injury in a primate model. Am J Obstet Gynecol. 2012;207(6):475.e1-475.e14.

12. Gray DJ, Robinson HB, Malone J, et al. Adverse outcome in pregnancy following amniotic fluid isolation of Ureaplasma urealyticum. Prenat Diagn. 1992;12(2):111-117. 